\title{
Magneto-impedance effect in high permeability NiFeMo permalloy wires
}

M. Vázquez, a) J. M. García-Beneytez, and J. P. Sinnecker

Insitituto de Magnetismo Aplicado-UCM-RENFE and Instituto de Ciencia de Materiales-CSIC, P.O. Box 155, 28230 Las Rozas, Madrid, Spain

Lin $\mathrm{Li}^{\mathrm{b})}$

Carpenter Technology Corporation, Reading, Pennsylvania 19612

The giant magneto-impedance effect (GMI) has been measured in commercial HyMu80® permalloy wires, submitted to different treatments (annealing and cold-drawing). Although hysteresis measurements show quasibistable magnetic behavior, GMI variations as high as $160 \%$ can be found in the frequency range $1-10 \mathrm{MHz}$. The frequency dependence of the GMI is also analyzed in terms of a nonhomogeneous anisotropy distribution. (c) 1998 American Institute of Physics.

[S0021-8979(98)24411-2]

\section{INTRODUCTION}

The change in the high frequency impedance of very soft ferromagnetic materials upon the action of external dc fields, has been intensively studied over the last few years. ${ }^{1-3}$ This effect, known as magneto-impedance (MI) is attractive for technological applications, as it combines the appearance of large changes of the impedance ratio for applied fields of few $\mathrm{kA} / \mathrm{m}$ in strength, ${ }^{2}$ opposite to magnetoresistance effect, ${ }^{4}$ where large fields are required to achieve very small variations of the resistance. The giant magneto-impedance (GMI) effect is interpreted as arising from a classical electrodynamic origin $^{2,3}$ depending on the interaction between the magnetic field created by the ac current flowing through the sample and its magnetic domain structure. When the frequency of the current becomes high enough, the skin effect plays an important role, ${ }^{5}$ diminishing the effective section of the conductor and thus increasing its impedance. As the skin effect depends on the circular magnetic permeability, the sensitivity of the impedance of the sample to the action of an external field can be explained in terms of the variations of this circular permeability.

GMI has been measured in a wide range of materials, with particular interest focused on amorphous and nanocrystalline wires, ribbons, and films. ${ }^{6-8}$ The highest MI effect is observed for Co-rich amorphous wires, with nearly zero magnetostriction constant, where the internal stresses distribution created during quenching couple with magnetostriction to generate a relative small circular anisotropy and a circular domain pattern which enhances the value of the circular permeability. In this work we present measurements of the MI effect in commercially produced wires made with Carpenter HyMu80® alloy $\left(\mathrm{Ni}_{80} \mathrm{Mo}_{4.2} \mathrm{Fe}_{\text {bal }}\right.$ permalloy). This magnetically soft alloy wire exhibits quite interesting properties regarding its magnetization processes. ${ }^{9}$ MI variations as high as $150 \%$ are reported, and the evolution of MI ratio with different treatments is also analyzed.

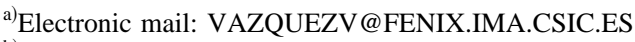

b)Present Address: YBM Magnex Inc., 110 Terry Drive, Newtown, PA 18940.
}

\section{EXPERIMENT}

Magneto-impedance measurements have been carried out in commercial Carpenter HyMu80® wires (nominal composition $\mathrm{Ni}_{80} \mathrm{Mo}_{4.2} \mathrm{Fe}_{\text {bal }}$. ) Three different samples were used for the experiment, obtained submitting the initial ascast wire to different treatments. The initial as-cast sample is produced with a diameter of $140 \mu \mathrm{m}$, but does not show good soft magnetic properties and special treatments are then required in order to observe magneto-impedance effect. Sample A is obtained after annealing the as-cast sample at $925^{\circ} \mathrm{C}$ for $40 \mathrm{~min}$ in a dry $\mathrm{H}_{2}$ atmosphere, followed by fast cooling. The diameter of the wire can be reduced to $44 \mu \mathrm{m}$ if a cold-drawn process is carried out in the as-cast wire, leading to sample B. Further annealing of this sample is applied to obtain sample C. In each case, pieces of $20 \mathrm{~cm}$ length were chosen for the experiment.

Measurements were carried out in a specially designed experimental setup, similar to one described elsewhere. ${ }^{10} \mathrm{Im}$ pedance variations with respect to a dc-magnetic field are determined by measuring the voltage drop over the sample when an ac current is flowing. Frequency range analyzed sweeps from 1 to $10 \mathrm{MHz}$. The current intensity is kept constant and equal to $10 \mathrm{~mA}$ (rms value) by monitoring the voltage drop over a high frequency resistance connected in series with the sample. Data are acquired using a HP3589A Spectrum Analyzer. An external Helmholtz coil system is used to apply dc magnetic fields up to $11 \mathrm{kA} / \mathrm{m}$ parallel to the axis of the sample. The magneto-impedance ratio, which depends on the applied magnetic field, is defined as $\Delta Z / Z(H)=100 \times\left[Z(H)-Z\left(H_{\max }\right)\right] / Z\left(H_{\max }\right)$, where $H_{\max }$ is the maximum applied magnetic field. The system is computer controlled through a GPIB interface. ${ }^{10}$ Quasistatic hysteresis loops have been measured using a conventional induction technique.

\section{RESULTS AND DISCUSSION}

Sample A has a characteristic loop of a soft magnetic material with a coercivity of $15 \mathrm{~A} / \mathrm{m}$, while in sample B a significant hardening was observed, with an increase of the coercive field up to $400 \mathrm{~A} / \mathrm{m}$ as a consequence of the cold 


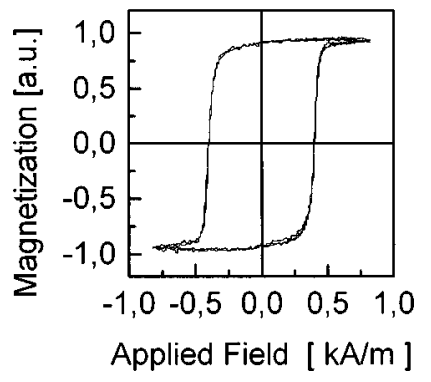

FIG. 1. Quasistatic axial hysteresis loops for cold-drawn sample (sample B).

drawing process that induces quite strong stresses in the sample. Figure 1 shows the hysteresis loop measured in this sample. Further annealing on sample $\mathrm{B}$ results in a decrease of the coercivity to a value of $30 \mathrm{~A} / \mathrm{m}$ in sample $\mathrm{C}$ as a consequence of the significant relief of the internal stresses. A common feature of all the loops (samples A, B, and C) is their quasibistable magnetic behavior: there is a critical field below which it is not possible to observe remagnetization. Above this field, hysteresis loops appear, as shown in Fig. 1. This behavior, typical of amorphous wires, can be explained considering that the main contribution to the magnetization process arises from domain wall motion.

GMI measurements for samples B and C are shown in Fig. 2, where the relative variation in the impedance, $\Delta Z / Z$, is plotted as a function of the applied magnetic field. Different curves correspond to different frequencies of the ac current, in the range from 1 to $10 \mathrm{MHz}$. The general trend is the decrease of $\Delta Z / Z$ for increasing dc field as a consequence of the decrease of the circular permeability. ${ }^{2}$

The largest MI ratio of $150 \%$ is found for sample C, at a frequency of $3 \mathrm{MHz}$, while the maximum variation of sample B is of $50 \%$ and takes place at a higher frequency of $7 \mathrm{MHz}$. Results for sample A (not shown) are very similar to those of sample C. For this sample, a maximum value of $130 \%$ is found at $1 \mathrm{MHz}$.

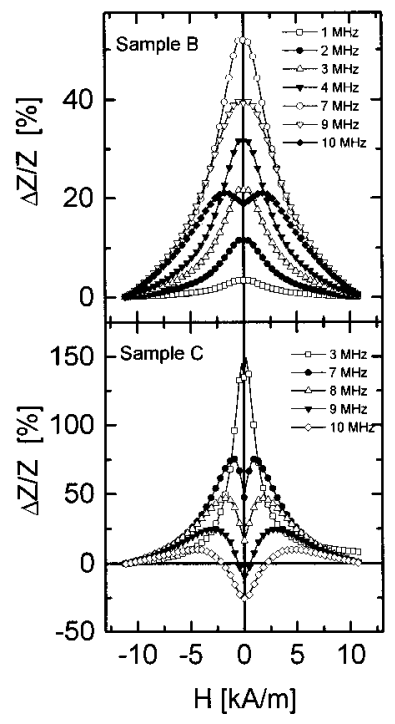

FIG. 2. Variations of the impedance of samples B (a) and C (b) with axial magnetic field for different frequencies.

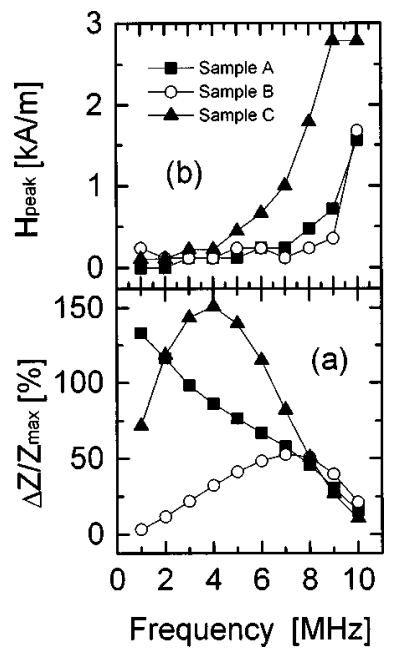

FIG. 3. (a) Evolution with frequency of the maximum value of MI ratio with the frequency of the ac current and (b) evolution of the magnetic field $H_{\text {peak }}$ where the maximum of the MI ratio takes place as a function of the frequency of the current.

The fact that sample B shows a smaller GMI ratio than samples $\mathrm{A}$ and $\mathrm{C}$ can be explained in terms of the relative magnetic softness of each sample. It is anyway remarkable the large magneto-impedance effect of sample B in spite of its much larger coercivity. As is well known, MI depends on variations of the circular permeability induced by external axial fields. These changes modify the penetration depth of the ac current and then the impedance of the sample. A softer magnetic material will, in general, present a higher variation of the circular permeability.

The evolution of the field dependence of the impedance with frequency is a common feature of all measurements performed. The maximum value of MI ratio changes with the frequency of the ac current as shown in Fig. 3(a). Samples B and $\mathrm{C}$ show a maximum located at 4 and $7 \mathrm{MHz}$, respectively, while sample A reaches its maximum for the lower frequency considered.

The magnetic field where the maximum value of the impedance is measured $\left(H_{\text {peak }}\right)$ also changes with frequency. The initial peak, located at $H=0$ (for sample A), evolves into two symmetric peaks which tend to move towards higher values as the frequency increases. The same tendency has been observed for the three samples under consideration. This effect can be seen in Fig. 3(b).

The maximum in the MI ratio is usually explained in terms of the existence of a circular anisotropy. For a fixed value of the external field (equal to the anisotropy field), the anisotropy is compensated, and a maximum in the permeability (and then in the MI ratio) is expected. The observation of this maximum in wires with a bistable behavior indicates that domain wall motion cannot be the only mechanism taking part in the magnetization process, as could be deduced from the shape of the hysteresis loops (Fig. 1). Instead, magnetization rotation from the circular to the longitudinal direction must be considered, as pointed out by Atkinson and Squire, ${ }^{11}$ in order to account for a variation in the circular permeability of the sample. 
On the other side, the shift of the maximum towards higher fields as the frequency increases [Fig. 3(b)] can be explained considering the existence of a radial anisotropy profile in the wire. An increase in the frequency is associated with a decrease in the penetration depth of the ac current through the sample. Then, for very high frequencies, the current is flowing only through a layer close to the surface of the material. As the transport properties (impedance in this case) depend on the behavior of this region of the material, the measurement of the impedance allows us to get information of the region close to the surface. The observed increase with frequency of the field where MI ratio is maximum (the circular anisotropy field) can be explained considering that the anisotropy is not homogeneous in all the wire section, but increases as one approaches the surface. As the local anisotropy is higher, the peak moves toward higher values. For very high frequencies, the anisotropy can be so large that the maximum applied field is not enough to saturate the sample. In this case, the impedance can have a smaller value for $H$ $=0$ than for $H=11 \mathrm{kA} / \mathrm{m}$. This is the case observed for sample $\mathrm{C}$ at $10 \mathrm{MHz}$.

\section{CONCLUSION}

High values of MI are reported for a Ni-rich permalloy wire presenting a quasibistable behavior. The analysis of the frequency evolution of the curves reveals the existence of a radial profile of anisotropies, and proposes MI measurements as a useful tool to study the magnetic behavior of regions close to the surface of the wire.

\section{ACKNOWLEDGMENTS}

The financial support of the Spanish CICYT under project MAT95/0273 is acknowledged. J. P. Sinnecker thanks the Brazilian agency FAPESP for the post-doctoral fellowship.

${ }^{1}$ K. Mohri, T. Kohzawa, K. Kawashima, H. Yoshida, and L. V. Panina, IEEE Trans. Magn. 28, 3150 (1992).

${ }^{2}$ L. V. Panina, K. Mohri, K. Bushida, and M. Noda, J. Appl. Phys. 76, 6198 (1994).

${ }^{3}$ R. S. Beach and A. E. Berkowitz, Appl. Phys. Lett. 64, 3652 (1992).

${ }^{4}$ M. N. Baibich, J. M. Broto, A. Fert, F. Nguyen van Dau, F. Petroff, P. Etienne, G. Creuzet, A. Fiederich, and J. Chazeles, Phys. Rev. Lett. 61, 2472 (1988)

${ }^{5}$ Landau and Lifshitz, Electrodynamics of Continuous Media (Pergamon, Oxford, 1975), p. 195

${ }^{6}$ F. L. A. Machado, C. S. Martins, and S. M. Rezende, Phys. Rev. B 51, 3926 (1995).

${ }^{7}$ J. Velázquez, M. Vázquez, D. X. Chen, and A. Hernando, Phys. Rev. B 50, 16737 (1994).

${ }^{8}$ R. L. Sommer and C. L. Chien, Appl. Phys. Lett. 67, 3346 (1995).

${ }^{9}$ L. Li, J. Appl. Phys. 81, 4287 (1997).

${ }^{10}$ J. P. Sinnecker, M. Knobel, M. L. Sartorelli, J. Shonmaker, and F. C. S. Silva, Proceedings of the Soft Magnetic Materials Conference SMM13, Grenoble, Sept. 1997.

${ }^{11}$ D. Atkinson and P. T. Squire, IEEE Trans. Magn. 33, 3364 (1997). 